\title{
Time and dose dependency of bone-sarcomas in patients injected with radium-224,
}

\author{
D. Chmelevsky ${ }^{1}$, A.M. Kellerer ${ }^{2}$, C.E. Land ${ }^{3}$, C.W. Mays ${ }^{3}$, and \\ H. Spiess ${ }^{4}$ \\ ${ }^{1}$ Gesellschaft für Strahlen- und Umweltforschung (GSF) München, \\ Institut für Strahlenschutz, Ingolstädter Landstrasse 1, D-8042 Neuherberg, \\ Federal Republic of Germany \\ ${ }^{2}$ Institut für Medizinische Strahlenkunde, Universität Würzburg, \\ Versbacher Strasse 5, D-8700 Würzburg, Federal Republic of Germany \\ ${ }^{3}$ National Cancer Institute, Radiation Epidemiology Branch, Landow 3A22, \\ Bethesda, MD 20892, USA \\ ${ }^{4}$ Kinderpoliklinik Universität München, Pettenkoferstrasse 8a, D-8000 München 2, \\ Federal Republic of Germany
}

Received December 7, 1987 / Accepted in revised form December 23, 1987

\begin{abstract}
Summary. The time course and dose dependency of the incidence of bone-sarcomas among 900 German patients treated with high doses of radium-224 is analysed in terms of a proportional hazards model with a log-normal dependency of time to tumor and a linear-quadratic dose relation. The deduced dose dependency agrees well with a previous analysis in terms of a non-parametric proportional hazards model, and confirms the temporal distribution which has been used in the Radioepidemiological Tables of NIH. However, the linear-quadratic dose-response model gives a risk estimate for low doses which is somewhat less than half that obtained under the assumption of linearity.
\end{abstract}

\section{Introduction}

For more than three decades, a follow-up has been conducted of patients who were injected with high doses of the short lived $\alpha$-emitter radium-224 in several German clinics in the years 1944 to about 1964. This is a group of 218 children and juveniles, mostly treated for bone tuberculosis with activities up to $5 \mathrm{MBq}$ per $\mathrm{kg}$ body weight, and 682 adults treated for ankylosing spondylitis or bone tuberculosis or other diseases with, on the average, substantially lower activities per body weight. The observations, published in a series of earlier reports $[6,7,8]$, have become one of the most important sources of knowledge on the dose and time dependency of bone sarcomas induced by an $\alpha$-emitter. They continue to provide essential information

* Dedicated to Prof. W. Jacobi on the occasion of his 60th birthday

** Work performed under Euratom contracts BI6-D-083-D, BI6-F-111-D, U.S. Department of Energy contract DE-AC02-76 EV-00119, the U.S. National Cancer Institute 
also on other late effects such as cataracts, tooth breakages, or severe kidney damages [8].

The bone sarcomas in the radium-224 patients occurred in a wave which reached a maximum about 8 years after treatment and continued, at decreasing level, to about 30 years [6]. This type of temporal distribution is strikingly similar to that of the leukemias induced in the survivors of the atomic bomb explosions in Hiroshima and Nagasaki [3].

The dose and time dependencies of radiation induced malignancies are critical input information for the establishment of radioepidemiological tables. The tables established by NIH [9] specify the probabilities of causation of various tumors. The only effect of an incorporated $\alpha$-emitter considered in these tables is that of bone sarcomas induced by radium-224; the treatment is based on the follow-up of the radium-224 patients. In the mathematical model a log-normal temporal distribution of the incidence of bone sarcomas after exposure to radium-224 is assumed. The parameters of this distribution and an assumed linear dose dependency are obtained from simplified calculations, with no differentiation between dose classes and with approximative corrections for competing risks, i.e. for the disappearance of patients from the observation. In a more recent study Land [4] extended this treatment and has given somewhat modified results, again in terms of a lognormal distribution of times to the tumor. He also compared the essential results with temporal distributions for other radiation induced tumors.

A somewhat different approach, in terms of a non-parametric maximum likelihood fit to a proportional hazards model [1], was used in an analysis of the time and dose distribution of the incidence of bone sarcomas in the radium-224 patients. This study was not restricted to the assumption of a linear dependency on dose, and the fit of the data to a linear-quadratic dose relation resulted in substantially reduced risk estimates at low doses. The time dependency appeared to be independent of dose and the age at treatment.

The singular nature of the data and their implications for the probability of causation tables make it desirable to combine the treatment in terms of the log-normal distribution of times to the tumor with a proportional hazards analysis.

\section{Summary of the data}

The follow-up of the radium-224 patients comprises observations on various late effects, apart from the bone sarcomas, but only the latter data are considered in the present context. The data are summarized in Table 1. Additional details are found in several earlier reports [5-8]. The classification in the table follows the one adopted earlier by Spiess and Mays. Among 900 patients, 38 with unknown doses (among them 6 with bone sarcoma) were excluded from the analysis. Of the 48 patients with known doses who incurred bone sarcomas, 2 later developed a further bone sarcoma. In both cases the pathology indicated that these were no secondary tumors, and the two additional cases were, therefore, included in the analysis. The 


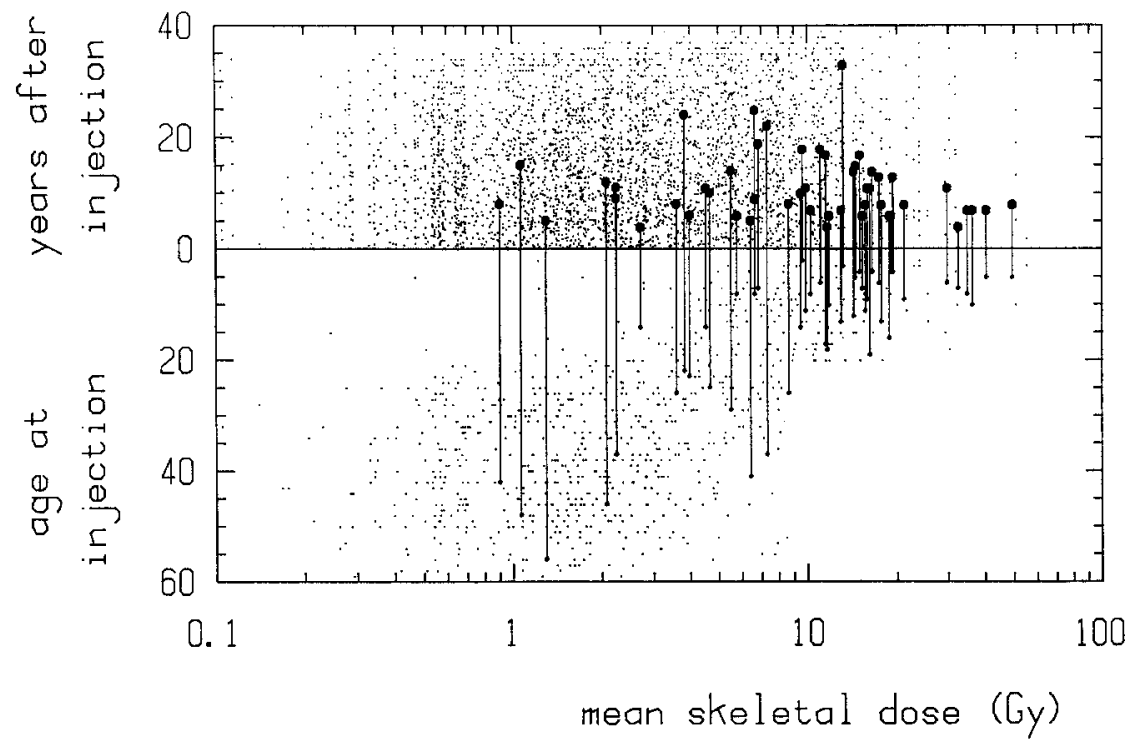

Fig. 1. The 48 patients who incurred bone sarcoma are represented by vertical lines from their age at injection to time at tumor diagnosis. The 50 bone sarcomas are given by heavy dots. The light dots in the lower panel give the distribution of ages at injection. The patients without bone sarcoma are represented, in the upper panel, by a chain of points from year at injection to last year of follow-up, with one point on the average per 5 years

decision had, however, no substantial influence on the results; these remain essentially unchanged, when the analysis was restricted to first bone tumors i.e., when patients were considered at risk only until a bone tumor occurred. The individual mean skeletal doses ${ }^{1}$ were calculated by Mays on the basis of the injected activities, body weights, and ages at treatment of the patients [10].

Figure 1 complements Table 1 . It represents the distribution of the patients in mean skeletal dose. In the bottom part of the diagram, each patient is represented by his age at treatment with a small dot. In the top part of the diagram all patients without bone sarcoma are represented by a vertical chain of dots, somewhat randomly spaced but with one dot on average for 5 years of observation. The patients who developed a bone sarcoma are indicated by solid vertical lines. The position of the heavy dots, which represent the appearance of bone sarcomas, can be related to the density of neighbouring points; this indicates the number of patients at risk with similar doses at similar times after treatment.

In the bottom part of the diagram, one distinguishes readily the group of adults with their lower doses from the group of children and juveniles with higher doses. In the upper part of the diagram one notes the increasing

\footnotetext{
${ }^{1}$ In the present article numerical results are given in terms of the 'mean skeletal dose', $D_{s}$. Alternatively one could use the quantity 'endosteal dose', $D_{e}$, which is the reference quantity in the BEIR report [2] and in the Radioepidemiological Tables [9]
}

$$
D_{e}=7.5 D_{\mathrm{s}}
$$


Table 1. Synopsis of data through 1987

Number of patients included in analysis $=832$; Number of patients with bone sarcoma $=48$ (double: 2); Number of patients excluded from analysis because of missing dose $=38$ (6 with bone sarcoma); lost to follow up $=30$

\begin{tabular}{|c|c|c|c|c|c|c|}
\hline & \multicolumn{2}{|c|}{ Number of } & \multicolumn{3}{|l|}{ Mean age $^{a}$} & \multirow{2}{*}{$\begin{array}{l}\text { Mean } \\
\text { skeletal } \\
\text { dose }(G y)^{2}\end{array}$} \\
\hline & patients & $\begin{array}{l}\text { with } \\
\text { bone } \\
\text { sarcoma }\end{array}$ & $\begin{array}{l}\text { at first } \\
\text { injection }\end{array}$ & $\begin{array}{l}\text { at last } \\
\text { follow up }\end{array}$ & $\begin{array}{l}\text { at bone } \\
\text { sarcoma }\end{array}$ & \\
\hline all adults & 628 & 13 & $38.7(10.8)$ & $61.8(11.6)$ & $46.5(10.9)$ & $2.08(1.50)$ \\
\hline ankylosing spondylitis & 366 & 5 & $41.3 \quad(9.5)$ & $63.7(10.1)$ & $58.2 \quad(4.4)$ & $1.43(1.00)$ \\
\hline tuberculosis & 218 & 8 & $33.5(10.6)$ & $58.8(13.0)$ & $39.1 \quad(6.4)$ & $3.25(2.22)$ \\
\hline other & 44 & 0 & $43.1(12.6)$ & $61.0(13.5)$ & $-\quad(-)$ & $1.73(1.26)$ \\
\hline male adults & 469 & 11 & $39.3(10.2)$ & $62.3(11.1)$ & $47.6(11.2)$ & $1.85(1.52)$ \\
\hline ankylosing spondylitis & 345 & 5 & $41.2 \quad(9.4)$ & $63.6(10.1)$ & $58.2(4.4)$ & $1.42(1.00)$ \\
\hline tuberculosis & 104 & 6 & $32.7 \quad(9.7)$ & $58.4(12.4)$ & $38.8 \quad(6.5)$ & $3.25(2.03)$ \\
\hline other & 20 & 0 & $40.5(10.8)$ & $58.7(13.6)$ & $(-)$ & $1.93(1.41)$ \\
\hline female adults & 159 & 2 & $37.0(12.4)$ & $60.4(13.1)$ & $40.0 \quad(6.0)$ & $2.78(2.23)$ \\
\hline ankylosing spondylitis & 21 & 0 & $42.5(10.7)$ & $64.7 \quad(8.5)$ & (8) & $1.62(0.98)$ \\
\hline tuberculosis & 114 & 2 & $34.2(11.2)$ & $59.1(13.5)$ & $40.0 \quad(6.0)$ & $3.25(2.39)$ \\
\hline other & 24 & 0 & $45.3(13.5)$ & $62.9(13.1)$ & $-\quad(-)$ & $1.57(1.10)$ \\
\hline children and juveniles & 204 & 35 & $11.6 \quad(5.3)$ & $38.5(13.1)$ & $19.7 \quad(5.8)$ & $10.71(8.82)$ \\
\hline boys & 105 & 16 & $11.6 \quad(5.3)$ & $39.5(12.9)$ & $22.2 \quad(6.4)$ & $11.36(9.61)$ \\
\hline girls & 99 & 19 & $11.7 \quad(5.3)$ & $37.3(13.3)$ & $17.7 \quad(4.4)$ & $10.02(7.84)$ \\
\hline
\end{tabular}

${ }^{a}$ Mean and (standard deviation)

frequency of bone sarcomas in the high dose class. One concludes, even from this diagram, that there is no appreciable shift in the average time to the bone sarcomas with increasing dose.

\section{Summary of results from earlier analyses}

Results from the continuing follow-up of the radium-224 patients were reported in a number of earlier articles [6-8]. Some essentials are summarized in the following.

Based on national bone-sarcoma rates, the expected number of bone sarcomas was, for the follow-up until 1984 only 0.2 [8]. This number is so small, in comparison with the 50 bone sarcomas observed in this group of patients, that little error can be introduced by the assumption that all of the observed cases were induced by radiation. Of these bone sarcomas all but one occurred from 4 to 25 years after the radium-224 treatment. The last bone sarcoma occurred recently, 33 years after the radium-224 injections. Before this observation, it had been believed that the enhanced bone-sarcoma rate was mainly extinguished, 25 years after injection. The top panel of Fig. 2 gives the distribution, in time after treatment, of the absolute numbers of observed bone sarcomas.

The same panel also contains the number of patients still in the follow-up at specified times after treatment. The time of observation is more than 
Fig. 2. In the upper panel the numbers of bone sarcomas and the numbers of patients still under observation are plotted versus time after injection.

The second panel from top gives the average of the mean skeletal dose in the collective of patients still at risk. The shaded area represents the standard error. The second panel from bottom gives the cumulative rate per gray that results under the assumption of linearity in dose (see (2)). The shaded area gives the standard deviation. The bottom panel gives the rate of bone sarcoma per year and per gray that corresponds to the cumulative rate in the second panel from bottom
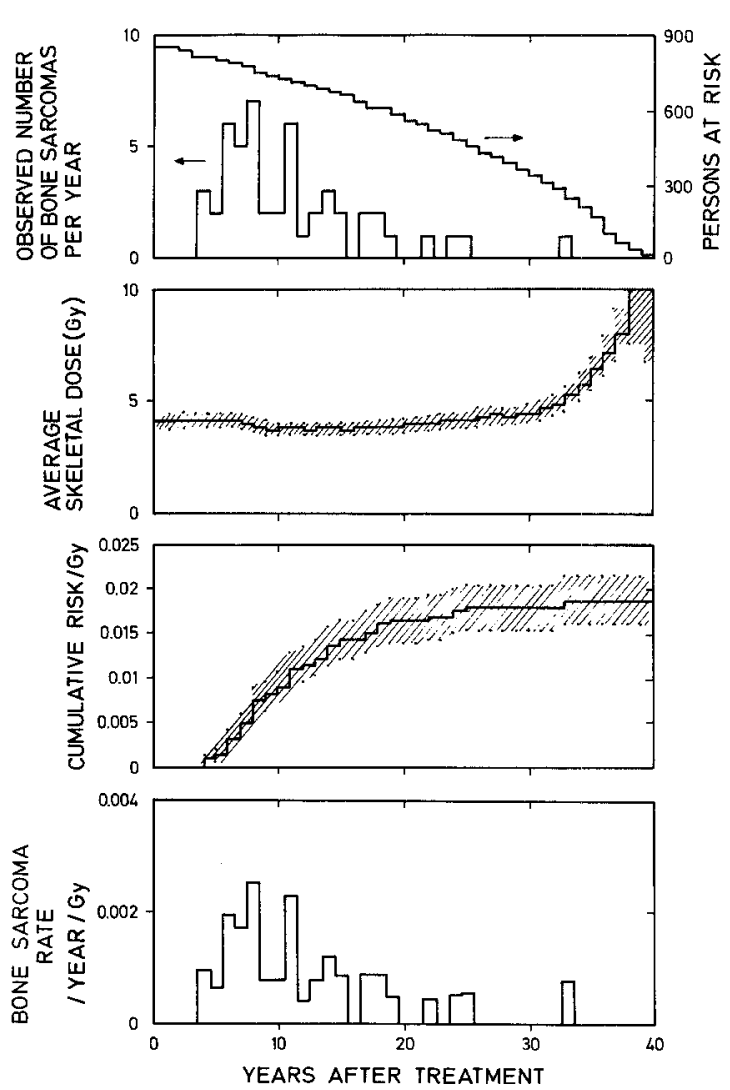

30 years for most of the surviving patients; for longer observation times the data can, therefore, still change.

\subsection{Sum-limit estimate and correction for changes in average dose}

From a competing risk corrected analysis - and under the assumption of proportionality of risk and dose - Mays and Spiess concluded that there are no substantial differences in the bone-sarcoma rates in the different groups of patients $[6,8]$. Neither age at injection, nor original illness, nor sex appear to influence the rate of radiation induced bone sarcoma per unit dose. The data for the various groups of patients were, therefore, pooled, and - under the assumption of proportionality of risk and dose - the rate of bone sarcomas per unit dose was derived in its dependency on time after treatment.

Two basic quantities need to be defined for an explanation of the computations. The (differential) bone-sarcoma rate ${ }^{2}, r(t, D)$, refers to a patient who received the dose $D$ and who is still at risk at time $t$ after the treatment. If time is counted in years after treatment, $r(t, D)$ is the conditional probabili-

\footnotetext{
${ }^{2}$ The more technical term 'hazard function' is frequently used for the quantity, $r(t, D)$, and the term 'cumulative hazard function' for $R(t, D)$
} 
ty - given survival to that time - that a patient who received the dose $D$, incurs a bone sarcoma in the year $t$. A related quantity is the cumulative rate of bone sarcomas, which equals the integral of the rate, $r(t, D)$, and is denoted by $R(t, D)$. It is often practical to refer to the cumulative rate because it is less affected by statistical fluctuations.

If all patients are considered as members of one group without regard to their different doses, one can derive the cumulative rate from the sum-limit estimate:

$$
R(t)=\sum_{i \leqq t} \frac{n_{i}}{N_{i}}
$$

where $n_{i}$ is the number of bone sarcomas in year $i$ after treatment, and $N_{i}$ is the number of patients still under observation in this year.

The summation extends up to year $t$. Assuming a linear dependency on dose, one can divide $R(t)$ by the average dose, $\bar{D}$, in the collective, to obtain the cumulative rate per unit dose. This was the procedure in the earlier analysis [8]. The approach depends on the implicit assumption, that the average dose, $\bar{D}$, in the collective still under observation does not change during the follow-up. As seen in the second panel from top in Fig. 2, this assumption is largely met, at least in the first 30 years of the follow-up; the simplified analysis is, therefore, essentially correct.

It is nevertheless of interest to consider the more rigorous solution. If the average doses, $\bar{D}_{i}$, during the follow-up are not the same, one has again with a linear model - the following relation for the cumulative rate, $R(t) / \bar{D}$, at unit dose:

$$
R(t) / \bar{D}=\sum_{i \leqq t} \frac{n_{i}}{N_{i} \bar{D}_{i}}
$$

This formula has been used to derive the cumulative and the differential risks represented in the two lower panels of Fig. 2. The results are nearly identical with the ones obtained earlier [8].

In spite of its irregularities the temporal distribution in the bottom panel of Fig. 2 is in evident general agreement with an assumed log-normal distribution. This will be substantiated and quantified in the subsequent considerations.

First it will, however, be necessary to consider computations not based on a linear dose dependency.

\subsection{Computation in terms of a non-linear dose dependency}

An essential feature of the data - and an implicit condition in the earlier analysis by Mays and Spiess $[5,8]$ - is the absence of a recognizable correlation between time to tumor and dose. No systematic decrease of the mean time to the tumor with increasing dose is evident from Fig. 1, and no such decrease has been identified in computations performed with different age 
groups [1]. The apparent lack of dependence of time to the tumor on dose and age at exposure has made it possible to analyse the data in terms of a model that represents the excess tumor rate as the product of a base-line function, $r_{0}(t)$, depending merely on time after treatment, and a term, $h(D)$, depending on dose:

$$
r(t, D)=r_{0}(t) \cdot h(D)
$$

The cumulative rate follows the analogous equation:

$$
R(t, D)=R_{0}(t) \cdot h(D)
$$

where $R_{0}(t)$ is the cumulative of $r_{0}(t)$.

These relations correspond to a proportional hazards model. According to established statistical terminology, $r_{0}(t)$ is termed the base-line function. However, one must note the difference to another common usage where the term base-line function is utilized for the spontaneous, age specific tumor rate, and where the variable $t$ refers then to age, and not to time after radiation exposure. As stated, spontaneous rates can be disregarded in the present analysis, and $r_{0}(t)$ is not coupled to the age specific rates.

The proportional hazards computations can either be performed with no analytical expression postulated for the time dependency, i.e. for $r_{0}(t)$ (or $R_{0}(t)$ ), or they can be performed according to specified models, with assumed analytical expressions for the time and dose dependencies. The non-parametric analysis is more fundamental in the sense of avoiding possible bias of the results due to the assumption of an analytical expression for the time dependency. The parametric analysis is, on the other hand, less vulnerable to statistical fluctuations in the data, and it has the advantage to provide smooth dependencies which are more readily applicable to risk estimation, to the computation of probabilities of causation, or to modelling in terms of mathematical theories of radiation carcinogenesis.

The analysis has earlier been performed without an assumed analytical expression for $r_{0}(t)$ (or $R_{0}(t)$ ) but with the familiar linear-quadratic dependency on dose which is expressed by the equation:

$$
R(t, D)=R_{0}(t) \cdot\left(D+\beta D^{2}\right) \cdot \exp (-\gamma D) .
$$

The maximum likelihood solution of this model for the entirety of the data has provided the following relation for the asymptotic cumulative rate, i.e. the total risk:

$$
R(t \geqq 33, D)=0.0083 \cdot\left(D+0.23 D^{2}\right) \cdot \exp (-0.027 D)
$$

with the mean skeletal dose, $D$, in gray. Slight changes in the numerical values, compared to the earlier results [1], are due to the continuation of the follow-up till 1987 (see Table 2).

One may note that the assumed dose relation, whether linear or nonlinear, has little influence on the resulting base-line function. The temporal distribution in Fig. 3 agrees, therefore, closely with that obtained from the linear model (Fig. 2, two lower panels). 
Table 2. Dose parameters from the proportional hazards analyses

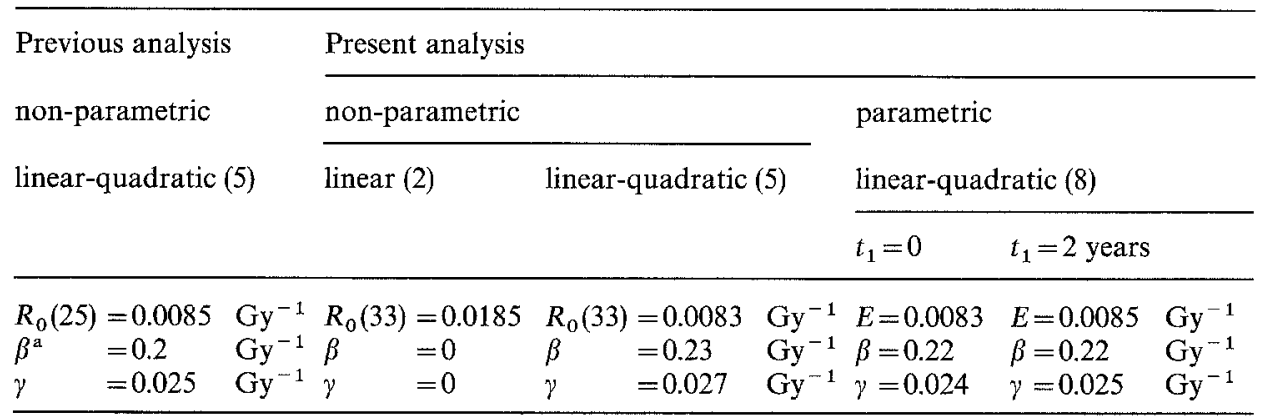

a The inverse of $\beta$ is the dose where the quadratic term equals the linear term

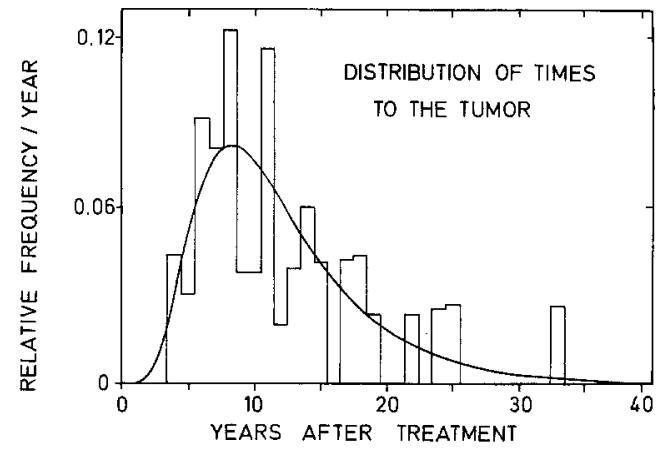

Fig. 3. The two base-line functions obtained with a proportional hazards analysis under the assumption of a linear-quadratic dose relation. The histogram results from the nonparametric analysis, the smooth curve from the analysis with a lognormal distribution of time to the tumor, with $t_{1}=0$ (see (8))

However, the result of the non-linear fit implies a substantially reduced risk estimate at small doses. The reduced estimate is in essential agreement with observations from a current follow up [12] of patients receiving the present day radium-224 therapy with considerably reduced doses. The changed risk estimate makes it desirable to perform the proportional hazards computations also in terms of a log-normal temporal distribution and to obtain, in this way, a comparison with the NIH results [9].

\section{Proportional hazards analysis in terms of a log-normal distribution of times to the tumor}

The proportional hazards model was used in the previous analysis to quantify the risk due to radium-224. To avoid possible bias, no analytical expression was used for the base-line function, $r_{0}(t)$. The disadvantage of the method is, that one obtains a discontinuous temporal distribution, as shown by the histogram in Fig. 3. For other applications, particularly for the computations of probabilities of causation, one requires smooth distributions. It is then desirable to perform a fully parametric analysis. As emphasized by Land [4], the radium-224 induced bone sarcomas share with the radiation induced leukemias the characteristic of wave like temporal distributions 
which reach a maximum several years after the exposure and become very small after a few decades. One speaks of an absolute risk model, since there is no assumed linkage between the age specific spontaneous incidence and the time dependency of the radiation induced malignancies ${ }^{3}$. The similarity is particularly close with the radiation induced chronic granulocytic leukemia; for this leukemia, too, it is assumed that the temporal distribution is independent of dose and, of age at exposure. Land [4] has proposed to fit such data with a log-normal distribution of times to the tumor:

$$
T(t)=\frac{1}{\sqrt{2 \pi} \sigma} \int_{t_{1}}^{t} \exp \left(\frac{-\left(\ln \left(\tau-t_{1}\right)-\ln \left(t_{0}\right)\right)^{2}}{2 \sigma^{2}}\right) \mathrm{d}(\ln (\tau))
$$

$T(t)$ is the cumulative distribution of appearance times, $t$; the notation $T(t)$ (rather than $R_{0}(t)$ ) is chosen in conformance with the notation in the NIHtables. $T(t)$ is independent of dose, and normalized to 1 . In order to obtain the cumulative bone-sarcoma rate, one has to multiply $T(t)$ by the total risk, at the specified dose, over the entire expression time. Equation (7) contains three parameters. $t_{0}$ and $\sigma$ are the two parameters of the log-normal distribution, and $t_{1}$ is an assumed minimum latent time, to account for a minimum manifestation period for bone sarcomas (or leukemias).

The analysis in terms of the log-normal distribution has been introduced by the NIH-tables committee [9] and by Land [4] who applied it to the leukemias among the atomic bomb survivors, the British ankylosing spondylitis patients treated by x-rays, and to the bone sarcomas in the radium-224 patients. The NIH panel derived the best fit parameters of the log-normal distribution for the bone sarcomas among the radium-224 patients in an approach based on reweighted least squares. They referred - as did Mays et al. [8] - the calculations merely to the number of patients still under observation at any time. As pointed out, this approach is valid for a linear model and under the condition that the average skeletal dose of those under observation changes little during the follow-up. The calculations were based on 898 patients, including those 38 (with 6 bone sarcomas) without known dose, who were excluded from the present analysis.

The time distribution used for the $\mathrm{NIH}$ tables is indicated in Fig. 4 (broken line). This is the derivative of the integral function in (7). The result is very close to the time dependency obtained in the proportional hazards computations to be described subsequently (solid curve in Figs. 3 and 4).

The importance of the fitted log-normal distribution for the probability of causation tables, and, generally, for quantification of dose and time dependencies of radiation induced cancers, justifies that a similar analysis be performed also in terms of the proportional hazards model which takes into account a non-linear dose dependency of the bone sarcomas. Accordingly

\footnotetext{
${ }^{3}$ For an alternative approach, in which a multiplicative linkage is assumed in analyses of both bone sarcoma and leukemia see [4]. In that paper, however, the application to bone sarcoma is flawed by a computational error resulting in an erroneously flat expected rate at zero dose (Land, personal communication)
} 


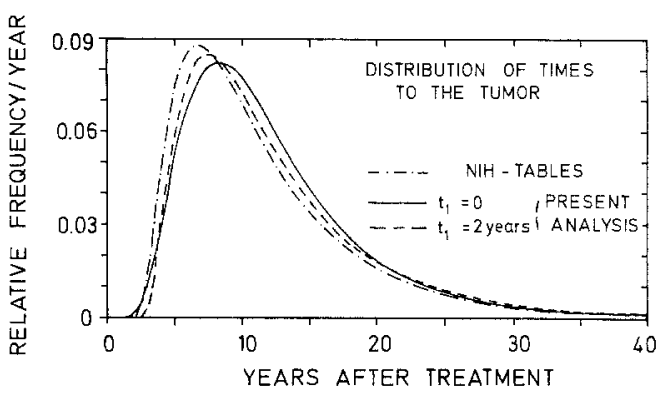

Fig. 4. The different log-normal distributions fitted to the times to bone sarcomas in the collective of radium-224 patients

the computations are performed with the linear-quadratic dose dependency:

$$
R(t, D)=T(t) \cdot E \cdot\left(D+\beta D^{2}\right) \cdot \exp (-\gamma D)
$$

In accordance with the notation in the NIH-tables, $E$ designates the total risk, i.e. the asymptotic value of the cumulative rate, when the dose dependent term equals unity. The difference to the earlier proportional hazards analysis is that the log-normal distribution, $T(t)$, takes the place of the non-parametric base-line function, $R_{0}(t)$.

Excluding negative values of the parameter $t_{1}$, one obtains the maximum likelihood with the parameters $t_{0}=10.7$ years and $\sigma=0.52$ and with $t_{1}=0$, i.e., with no minimum latent period. This solution is plotted as solid curve in Fig. 3 (and in Fig. 4). The absence of a minimum latent period may seem to be in contradiction with the fact that induced tumors can appear only after a minimal duration required for tumor growth. However, as seen from the figures, there is a virtual minimum latent period, because the amplitude of the time distribution is very small for times, $t$, less than about 2 years. This is a duration consistent with known cell kinetic parameters. It takes 30 volume-doubling times for a cancer cell, $10 \mu \mathrm{m}$ in diameter to enlarge to a detectable tumor, $1 \mathrm{~cm}$ in diameter. Sequential $\mathrm{x}$-ray measurements of tumor size [11] indicate an average volume doubling time of roughly one month for human osteosarcomas. Thus the average tumor growth time is approximately 30 months or 2.5 years. The shortest time to bone sarcoma diagnosis in the radium-224 patients was 3.5 years (rounded to 4 years).

Although the maximum likelihood solution with $t_{1}=0$ is consistent with a practical minimal latent period, it is nevertheless of interest to consider the optimal fit with an assumed latent period $t_{1}=2$ years which corresponds to the observations on tumor growth; the resulting solution is also given in Fig. 4 (dashed curve) and its parameters are listed in Tables 2 and 3. The two curves are in close agreement, and it is evident that the analysis can not decide against either solution. It is also seen that the results agree well with those in the NIH tables. The temporal distribution earlier deduced is, therefore, confirmed by the present analysis which accounts for the distribution of skeletal doses and for a non-linear dependency on dose. The close agreement is due to the fact that the distribution of mean skeletal doses among the patients remains substantially unchanged over most of the followup period (see Fig. 2). One notes, furthermore, that the inclusion of the patients with unknown doses has little influence on the results. 
Table 3. Parameters of the log-normal distributions. Parameters $\sigma, t_{0}$ and $t_{1}$ : see (7). $t_{\text {mod }}$ : most probable time to the tumor (see (9)); $t_{\text {med }}$ : median time to the tumor (see (10)); $\bar{t}$ : mean time to the tumor (see (11))

\begin{tabular}{rlll}
\hline & \multicolumn{2}{c}{ Present analysis } & NIH-parameters \\
\hline$\sigma=$ & 0.52 & 0.68 & 0.69 \\
$t_{0} \quad$ (years) $=$ & 10.7 & 8.5 & 8.3 \\
$t_{1} \quad$ (years) & 0 & 2.0 & 1.52 \\
$t_{\text {mod }}$ (years) $=$ & 8.2 & 7.4 & 6.7 \\
$t_{\text {med }}$ (years) $=$ & 10.7 & 10.5 & 9.8 \\
$t \quad$ (years) & $=12.3$ & 12.7 & 12.1 \\
\hline
\end{tabular}

In Fig. 3 the parametric temporal distribution is compared to the earliernon-parametric time dependence, $r_{0}(t)$. Table 2 gives the dose parameters for the non-parametric (2) and (5), and for the parametric (8) analyses. As is seen from the table, the differences in the derived assumed temporal distributions, have little influence on the dose parameters. The parametric analysis in terms of a linear quadratic dose relation confirms the earlier finding of a substantial quadratic term in dose, and of a risk coefficient at low doses (linear term) which is, compared to values in the NIH tables, reduced by a factor 2 .

The parameters of the temporal distributions obtained from the present analysis, with and without assumed minimum latent period, and those in the NIH tables are compared in Table 3 . One must note that these parameters refer to the log-normal distribution, so that $t_{0}$ is not the mean latent period and $\sigma$ is not the standard deviation of the latent periods. However one can give the relations for the more direct characteristics of the distributions, especially the equations for the modal time, $t_{\bmod }$, to the tumor (i.e. the most likely time), the median time, $t_{\text {med }}$, and the mean time, $\bar{t}$, to the tumor:

$$
\begin{aligned}
& t_{\text {mod }}=t_{0} \exp \left(-\sigma^{2}\right)+t_{1} \\
& t_{\text {med }}=t_{0}+t_{1} \\
& \bar{t}=t_{0} \exp \left(0.5 \sigma^{2}\right)+t_{1}
\end{aligned}
$$

For completeness, we state also the relations for the moments of the distributions of the times to the tumor and for the standard deviation, $\sigma_{t}$ (where the index $t$ is used to distinguish the quantity from the parameter $\sigma$ in the log-normal distribution):

$$
\begin{aligned}
& \bar{t}^{n}=t_{0}^{n} \exp \left(0.5 n^{2} \sigma^{2}\right) \\
& \sigma_{t}=t_{0} \sqrt{\left.\exp \left(\sigma^{2}\right)\left(\exp \left(\sigma^{2}\right)-1\right)\right)}
\end{aligned}
$$

\section{Conclusions and implications for the radioepidemiological tables}

The risk from radium-224 is - apart from the continued medical application - of minor practical importance. It is, nevertheless, of interest in the general 
context of the analysis of the dose and time dependencies of radiation induced cancers, especially by $\alpha$-emitters. The observations in the radium-224 patients have permitted the most precise determination of a time dependence among all relevant studies. The results have therefore model character for the formalism utilized in the Radioepidemiological Tables. The conclusions are closely related also to the formalism employed for leukemias.

The present analysis confirms the temporal distribution employed in the NIH tables. Variations ascribable to the use of slightly different data sets, different dose-response assumptions, and different minimal latent periods were minor. On the other hand, the dose-response model employed in this paper gives a low-dose risk estimate somewhat less than half that obtained under linearity, and would, if adopted, yield correspondingly smaller probability of causation values.

\section{References}

1. Chmelevsky D, Kellerer AM, Spiess H, Mays CW (1986) A proportional hazards analysis of bone sarcoma rates in German ${ }^{224} \mathrm{Ra}$ patients. In: Gössner W, Gerber GB, Hagen U, Luz A (eds) The radiobiology of Radium and Thorotrast. Urban \& Schwarzenberg, München, pp 32-37

2. Committee on the biological effects of ionizing radiations (1980) The effects on populations of exposure to low levels of ionizing radiation. National Academy Press, Washington, D.C.

3. Ichimuru H, Ishimuru T, Mikami M, Yamada Y, Ohkita T (1981) Incidence of leukemia in atomic bomb survivors and controls in a fixed cohort, Hiroshima and Nagasaki, October 1950-December 1978. Radiation Effects Research Foundation Technical Report 13-81. Hiroshima, Japan

4. Land CE (1987) Temporal distributions of risk for radiation-induced cancers. J Chron Dis 40 [Suppl 2]: $45 \mathrm{~S}-57 \mathrm{~S}$

5. Mays CW, Spiess H (1983) Epidemiological studies of German patients, injected with radium-224, Proceedings of the 16th Midyear Topical Meeting of the Health Physics Society. NTIS CONF 830101, Springfield, VA.: National Technical Information Service, Springfield, Virginia, pp 159-166

6. Mays CW, Spiess H (1984) Bone sarcomas in patients given radium-224. In: Boice JD, Fraumeni JF, (eds) Radiation carcinogenesis: epidemiology and biological significance: Raven Press, New York, pp 241-252

7. Mays CW, Spiess H, Gerspach A (1978) Skeletal effects following radium-224 injections into humans. Health Phys 35:83-90

8. Mays CW, Spiess H, Chmelevsky D, Kellerer AM (1986) Bone sarcoma cumulative tumor rates in patients injected with ${ }^{224} \mathrm{Ra}$. In: Gössner W, Gerber GB, Hagen U, Luz A (eds) The radiobiology of Radium and Thorotrast. Urban \& Schwarzenberg, München, pp 27-31

9. National Institutes of Health: Report of the NIH Ad Hoc Working Group to Develop Radioepidemiological Tables (1985) NIH Publication No. 85-2748. U.S. Department of Health and Human Services

10. Spiess H, Mays CW (1973) Protraction effect on bone sarcoma induction of ${ }^{224} \mathrm{Ra}$ in children and adults. In: Sanders CL, Busch RH, Ballou JE, Mahlum DD (eds) Radionuclide carcinogenesis. USAEC Symposium 29, CONF-720505. Springfield, VA.: National Technical Information Service Springfield, Virginia, pp 437-450

11. Spratt JS (1965) The rates of growth of skeletal sarcomas. Cancer 18:14-18

12. Wick RR, Chmelevsky D, Gössner W (1986) Radium-224 risk to bone and haematopoietic tissue in ankylosing spondylitis patients. In: Gössner W, Gerber GB, Hagen U, Luz A (eds) The radiobiology of Radium and Thorotrast. Urban \& Schwarzenberg, München, pp 38-44 